\title{
Ultrafast dynamics in unaligned MWCNTs decorated with metal nanoparticles
}

\author{
G Manzoni ${ }^{1}$, S Ponzoni ${ }^{1}$, G Galimberti ${ }^{1}$, M Scarselli ${ }^{2}$, O Pulci $^{2}$, L Camilli ${ }^{3}$, \\ L Matthes ${ }^{4}$, P Castrucci $^{2}$ and S Pagliara ${ }^{1}$ \\ ${ }^{1}$-LAMP and Dipartimento di Matematica e Fisica, Università Cattolica, 25121 Brescia, Italy \\ ${ }^{2}$ Dipartimento di Fisica, Università di Roma Tor Vergata, 00133 Roma, Italy \\ ${ }^{3}$ Department of Micro- and Nanotechnology, Technical University of Denmark, 2800 Kgs. Lyngby, \\ Denmark \\ ${ }^{4}$ Institut fur Festkopertheorie und optik, Friedrich Schiller Universitat, Max-Wien Platz 1, Jena, Germany \\ E-mail: stefania.pagliara@unicatt.it
}

Received 19 January 2016, revised 11 April 2016

Accepted for publication 13 April 2016

Published 5 May 2016

\begin{abstract}
The relaxation dynamics of unaligned multi-walled carbon nanotubes decorated with metallic nanoparticles have been studied by using transient optical measurements. The fast dynamics due to the short-lived free-charge carriers excited by the pump are not affected by the presence of nanoparticles. Conversely, a second long dynamics, absent in bare carbon nanotubes, appears only in the decorated samples. A combination of experiment and theory allows us to ascribe this long dynamics to relaxation channels involving electronic states localized at the tubenanoparticle interface.
\end{abstract}

Keywords: carbon nanotubes, time-resolved spectroscopy, hybrid systems

(Some figures may appear in colour only in the online journal)

\section{Introduction}

Deriving their optical properties from excitonic excited states, carbon nanotubes (CNTs) are strong absorbers of light from the near-infrared to the ultraviolet [1-3]. Moreover, like semiconductor nanocrystals, they exhibit an extremely efficient electron-electron scattering as well as an inefficient electron-phonon (particularly acoustic phonons) interaction [4-6] making them good candidates for the generation of multiple electron-hole pairs (MEG) [7].

In addition, due to their extraordinary electrical conductivity [8], CNTs provide quite efficient transport paths for carriers, which have a very high probability to reach electrodes and generate photocurrent in devices based on CNT photoexcitation processes. The bottleneck in CNT-based photovoltaic systems is the ultrafast recombination of the photogenerated charge carriers. However, the integration of CNTs into hybrid architectures shows an enhanced ability to trap electrons and reduce the ultrafast electron-hole recombination, which eventually provides a new way to modulate and improve the performance of CNT-based light-harvesting devices.
Among these hybrid systems, CNTs decorated with metal nanoparticles (NPs) have recently attracted much attention within a wide range of applications [9-11] including gas sensors [12-15] and catalysis [16] as well as optoelectronic and light-energy conversion devices [17-20].

In a previous paper of ours [21], the photo-response signal of multi-walled carbon nanotubes (MWCNTs) decorated with discrete metal NPs $(\mathrm{Au}, \mathrm{Ag}$, and $\mathrm{Cu}$ ) on the nanotube outer walls has been investigated. In particular, decorated MWCNTs exhibit a significant increase in the photocurrent with respect to that of bare tubes. From theoretical considerations, this increase has been ascribed to the presence of additional charges due to a static charge transfer from the NP towards the CNT at the interface. Nonetheless, it remains questionable whether the CNT-NP interface modifies only the quantity of available carriers in CNTs or even creates new excitation/relaxation channels.

In this work, in order to shed light on the processes at the basis of the photocurrent increase [21], we experimentally investigate in the time domain the excitation/relaxation channels of the carriers excited in decorated CNTs. 
Employing ultrafast time-resolved optical spectroscopy, we find an increase, with respect to the pristine CNTs, of the fast dynamics signal coming from the photoexcitation of the additional static charge. From the sign of the dynamics it is possible to assess that the free-electron-like character of these carriers is preserved in decorated as well as in pristine CNTs. Moreover, only in decorated CNTs, a second long dynamics ascribed to carriers excited in localized states at the CNT-NP interface appears, as confirmed by $a b$ initio calculations.

\section{Methods}

Time-resolved reflectivity measurements have been performed with an amplified $1 \mathrm{kHz}$ Ti:Sapphire laser system. It produces $150 \mathrm{fs}, 1.55 \mathrm{eV}$ light pulses, and permits us to excite the samples with pump fluence in the range $5-100 \mathrm{~mJ} \mathrm{~cm}^{-2}$. The transient response has been collected with the same probe photon energy $(1.55 \mathrm{eV})$, while the pump photon energy has been tuned in the visible-near-infrared (VIS-NIR, 1.80-2.03 $\mathrm{eV})$ thanks to an optical parametric amplifier and the harmonic generation of non-linear crystals. The pump fluence was kept to $11 \mathrm{~mJ} \mathrm{~cm}^{-2}$.

MWCNTs were grown on $\mathrm{Si}(111)$ substrate by the chemical vapor deposition process, using $\mathrm{Fe}$ as a catalyst. The growth was performed in a chamber operating in ultra-high vacuum (UHV) conditions (base pressure $10^{-8}$ Torr). Metal deposition ( $\mathrm{Au}, \mathrm{Ag}$ and $\mathrm{Cu}$ ) was performed in situ by thermal evaporation with the CNT samples kept at room temperature and under UHV conditions in the same chamber. The same deposition rate of $0.5 \AA \mathrm{min}^{-1}$ was used for all the samples [22].

Four samples have been considered for this study. One of them was of bare MWCNTs, the other three consisted of MWCNT films decorated with $0.5 \mathrm{~nm}$ of $\mathrm{Ag}, \mathrm{Au}$ and $\mathrm{Cu}$, respectively. The quality of the films has been deeply investigated as reported in [22].

For the theoretical approach, we have performed firstprinciples simulations within density functional theory (DFT) in the local density approximation (LDA) [23-26] using the ESPRESSO package [27]. Since the average diameter of our CNTs is quite large, we have simulated them using a single graphene layer. In addition, due to the high computational effort, the basic structural and electronic modifications of the graphene when decorated with $\mathrm{Cu}, \mathrm{Ag}$, and $\mathrm{Au}$ metal clusters have been computed for nanoparticle (NP) diameter up to $\sim 0.8 \mathrm{~nm}$. A graphene $9 \times 9 \times 1$ supercell (consisting of 162 $\mathrm{C}$ atoms) has been used for the largest NP. The geometry of the composite system was relaxed till the forces were less than $10^{-3}$ a.u. A set of up to $2 \times 2 \times 1 \mathrm{k}$-points was used to sample the Brillouin zone. The energy cut-off in the kinetic energy was $64 \mathrm{Ry}$. Optical properties of graphene decorated with metallic NPs where calculated within the random phase approximation (RPA). Since the size of the simulated NPs is smaller than the experimental one, we expect an overestimation of confinement effects (i.e. a blueshift) in the theoretical $\varepsilon_{2}$, which partially cancels with the redshift due to the use of DFT.

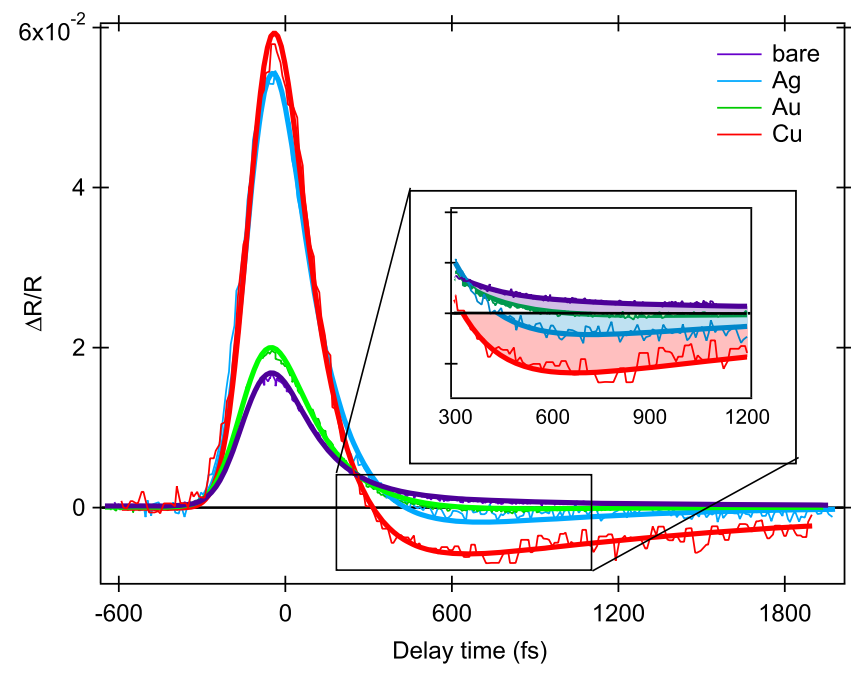

Figure 1. Comparison of the transient reflectivity spectra $\Delta R / R$ collected on the samples with a pump photon energy of $1.9 \mathrm{eV}$. In violet, the spectrum collected on the bare MWCNT, in light blue on $\mathrm{Ag}-\mathrm{MWCNTs}$, in green on Au-MWCNTs and in red on the $\mathrm{Cu}-$ MWCNT sample. In the inset, a zoom to point out the negative sign of the second dynamics in the decorated MWCNTs.

\section{Results and discussion}

Figure 1 shows the pump-probe measurements collected, on bare and decorated MWCNTs, with a pump photon energy of $h \nu=1.9 \mathrm{eV}$. While the sign of the transient response of the bare MWCNTs is positive for all the delay times, the signal of the decorated ones is positive at the beginning and becomes negative for longer delay times. The relaxation time of the positive first dynamics (150-200 fs) appears fast in both the bare and decorated samples.

The negative sign in reflectivity is the fingerprint of a photobleaching channel. The absorption of the pump pulse excites electrons into conduction bands creating holes in the valence bands. Until these carriers relax, transient filling effects on the final states decrease the absorption of the probe inducing a positive transient signal in transmittivity and negative in the absorption.

The $\Delta R / R$ signal, in principle, depends on both $\Delta \epsilon_{1} / \epsilon_{1}$ and $\Delta \epsilon_{2} / \epsilon_{2}$ where $\epsilon_{1}$ and $\epsilon_{2}$ are the real and the imaginary part of the dielectric function, respectively. For CNTs, similar to graphite, $\Delta R / R$ mainly depends on $\Delta \epsilon_{2} / \epsilon_{2}$ and then on the changes induced by the pump on the absorption of CNTs $[28,29]$. Therefore, when a photobleaching effect occurs, the reflectivity of the probe, behaving as the absorption, decreases and the transient reflectivity signal is negative [30].

For CNTs and graphitic systems in general, a photobleaching process is usually observed. Nevertheless, our unaligned MWCNT samples show a positive transient reflectivity. The same behavior has been reported for films of unaligned singled-walled carbon nanotube (SWCNT) bundles directly grown in UHV, not affected by other atomic species (e.g. contaminants, surfactants, molecules) [30]. In that case, we interpreted the positive response as the fingerprint of intraband transitions due to a Drude-like behavior of the 
carriers excited by the laser pump. Although a significant difference exists between the structure of the SWCNTs of reference [30] (bundled and unaligned) and the MWCNTs studied here (unaligned), the data suggest that the tube architecture alone is able in MWCNTs to modify the intertube interaction favouring the free-electron-like character of the photoexcited carriers.

The free-electron-like behavior characteristic of the unaligned nanotubes, as we can observe in figure 1, persists even when the tubes are decorated with metallic NPs. The positive fast response is preserved, in fact, in all the decorated MWCNTs studied here, suggesting that the presence of metallic NPs on the nanotube outermost walls does not affect the intertube interactions.

In addition to the fast free-electron-like positive response, a second negative and long dynamics, corresponding to a photobleaching phenomenon, appears but only in the decorated tubes (see figure 1). The absence of the the long negative dynamics in the bare MWCNTs and the dependence of its intensity on the kind of NP clearly suggest that it is a genuine response of the hybrid system made up of MWCNTs plus metallic NPs.

A complete overview of the transient reflectivity response for the decorated MWCNTs, collected by changing the pump photon energy in the $1.8-2.03 \mathrm{eV}$ range, is shown in figure 2 .

To quantify the differences observed in the relaxation dynamics of MWCNTs and decorated MWCNTs, all the transient reflectivity signals have been interpolated by two exponential functions, convoluted with a Gaussian curve representing the laser pulse. The results obtained from the fitting procedure are shown in figure 3.

In particular, figure 3(a) displays on the left the intensity of the first positive dynamics ascribed to the Drude-like behavior of the carriers excited by the laser pump for the bare and decorated MWCNTs, and on the right the average values reported in a bar histogram.

The intensity of the first dynamics for decorated tubes does not change significantly in the explored photon energy range, suggesting that effects due to the low-dimensionality of the NPs such as plasmonic excitations can be disregarded [31]. Furthermore, the intensity increases going from the bare MWCNTs towards the decorated MWCNTs. Notably, the first dynamics of MWCNTs decorated with Ag and Cu NPs results in greater intensity than the others. As reported in the literature [21], a static charge transfer from the NP to the CNT, caused by a not negligible difference in electronegativity between carbon and $\mathrm{Cu}, \mathrm{Ag}$, and $\mathrm{Au}$, is present at the interface of the hybrid architecture. The presence of a static charge transfer permits us to have a larger availability of charges in the occupied states that increases the intensity of the signal. In accordance with this interpretation, $\mathrm{Ag}$ and $\mathrm{Cu}$ being less electronegative (1.9 Pauling electronegativity) than $\mathrm{Au}$ (2.4) with respect to the $\mathrm{C}$ atom (2.5), an increase in the first dynamics intensity for MWCNTs decorated with $\mathrm{Cu}$ and $\mathrm{Ag}$ is expected.

The intensity of the negative second dynamics, characteristic of the decorated MWCNTs, is displayed in

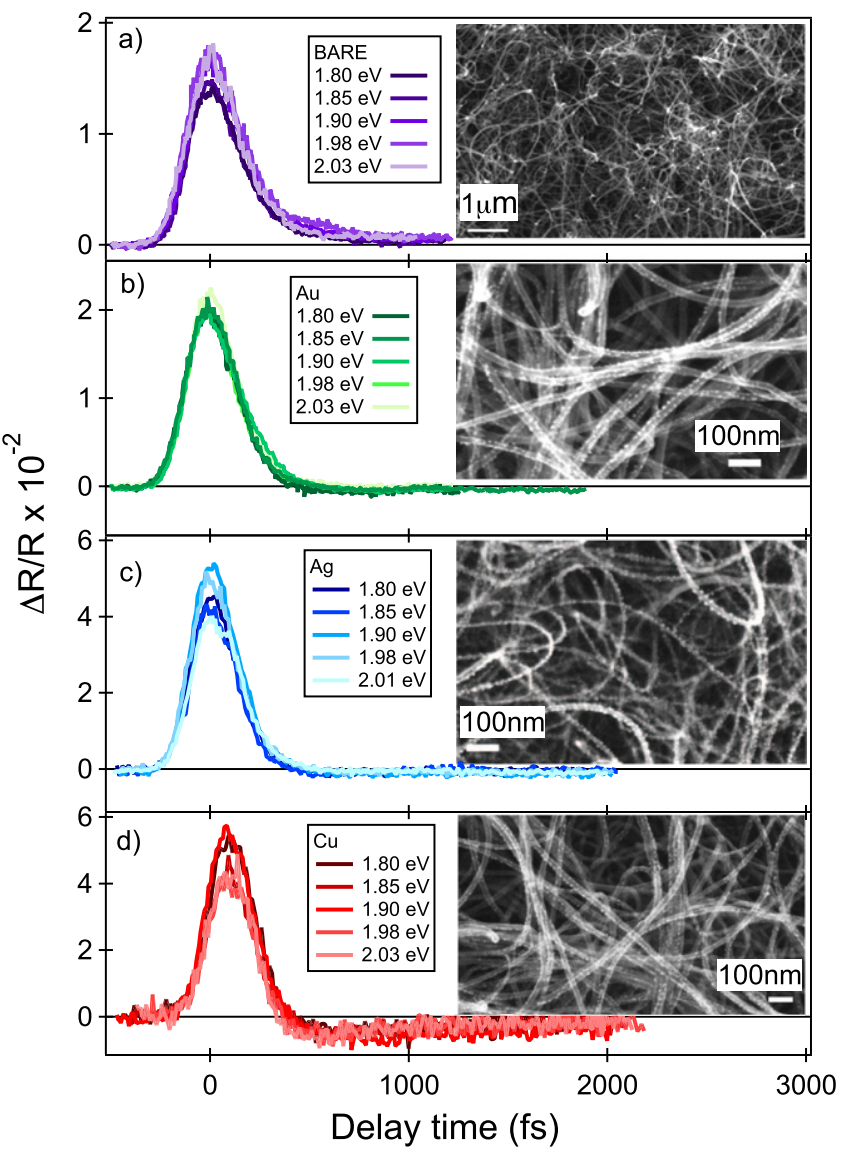

Figure 2. Transient reflectivity spectra collected on bare MWCNTs (a) and on MWCNTs decorated with $\mathrm{Au}$ (b), $\mathrm{Ag}$ (c) and $\mathrm{Cu}$ (d) nanoparticles. The spectra were obtained by changing the pump photon energy from $1.80 \mathrm{eV}$ to $2.03 \mathrm{eV}$, while the probe photon energy was kept equal to $1.55 \mathrm{eV}$. SEM image of a bare MWCNT (a), Au-MWCNT (b), Ag-MWCNT (c) and Cu-MWCNT sample (d) are also shown.

figure 3(b). As well as for the first dynamics, the intensity of this negative dynamics is larger for the MWCNTs decorated with $\mathrm{Cu}$ NPs. The relaxation times for both the first $\tau_{1}$ and the second $\tau_{2}$ dynamics are shown in figure 3(c). $\tau_{1}$ is comparable for all the studied MWCNTs, implying the occurrence of the same relaxation channel for the bare and decorated MWCNTs. This also confirms that the presence of NPs does not affect the free-electron-like character of the carriers excited in the unaligned tubes. On the other hand, the relaxation time of the negative second dynamics is longer for the $\mathrm{Cu}$-decorated MWCNTs (1.1 ps) with respect to the $\mathrm{Au}$ (0.4 ps) and Ag (0.6 ps) decorated samples.

In order to shed light on this second dynamics we have performed $a b$ initio simulations, within the DFT in the LDA of the electronic structure at the CNT/ NP interface. Due to the large diameter of the CNTs, the curvature of the tube could be safely neglected so that the NP/CNT interface was simulated using a graphene layer with a metal NP on top of about $0.8 \mathrm{~nm}$ diameter [21]. The dielectric function of decorated and pristine graphene has been calculated within the independent single-particle approach (Fermi golden rule). 


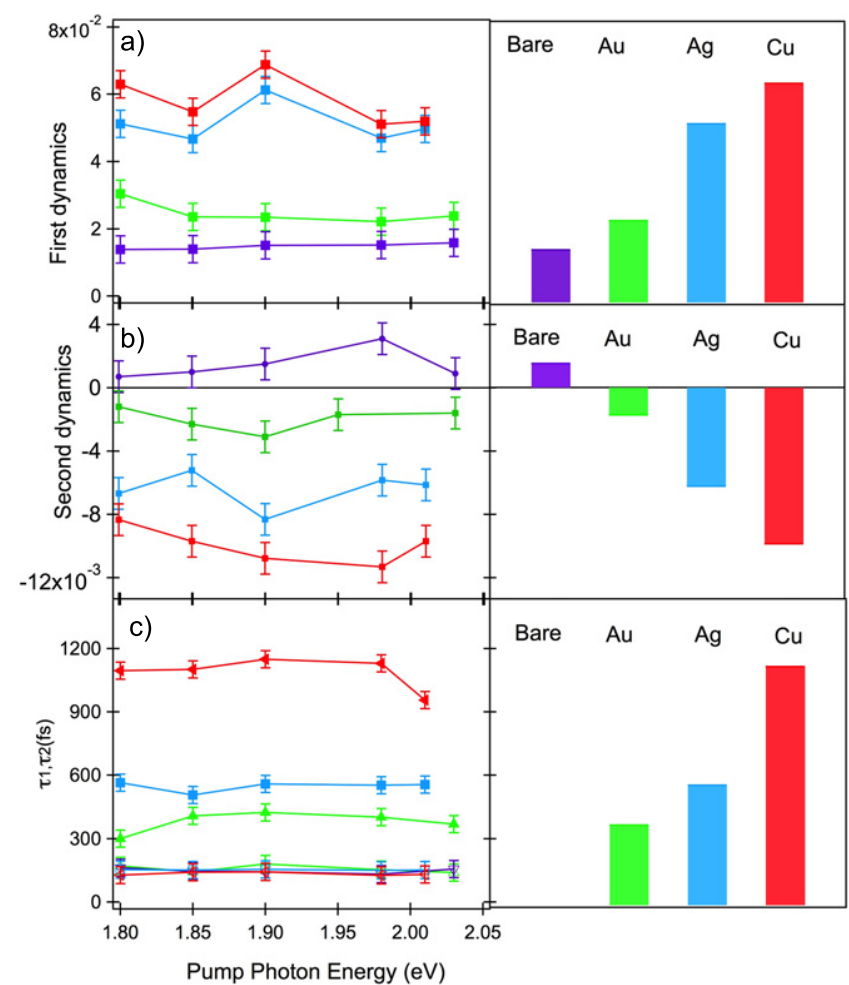

Figure 3. On the left the values obtained from the fitting procedure and on the right the average values of (a) the intensity of the first dynamics, (b) the intensity of the second dynamics and (c) the relaxation times of the first dynamics (empty markers) and of the second one (filled). The average relaxation times for the second dynamics are shown in the right panel of (c).

In figure 4 the calculated imaginary part of the dielectric function, $\epsilon_{2}$, is shown for both decorated and pristine graphene. A feature at about $1.8 \mathrm{eV}$, absent in pristine graphene, appears in decorated samples. This feature, clearly visible in graphene $/ \mathrm{Cu}$ and graphene $/ \mathrm{Ag}$ and less pronounced in the case of graphene/Au, originates from optical transitions involving states localized at the metal-graphene interface.

In the experiment reported here, by tuning the pump photon energy from 1.8 to $2.03 \mathrm{eV}$, the laser pump pulses are able to excite in a quasi-resonant way carriers in these localized interface states as well as carriers in excited states of the carbon nanotubes not affected by the presence of NPs. The result is that while the carriers excited in the electronic states of the CNTs give rise to the first fast dynamics whose positive sign is ascribed to the free-electron-like character, the carriers excited in the localized states at the NP/CNT interface, relaxing more slowly, are responsible for the second long dynamics. The negative sign of this second dynamics is consistent with a photobleaching process as expected for relaxation channels involving localized states.

In this scenario, it is plausible to assume that the significant increase in the photo-response signal measured on decorated CNT [21] is due not only to a static charge transfer, but to a photoinduced charge transfer originated by the presence of localized states at the interface.

Being the photo-response signal favored by the amount of the excited carriers together with the longest relaxation

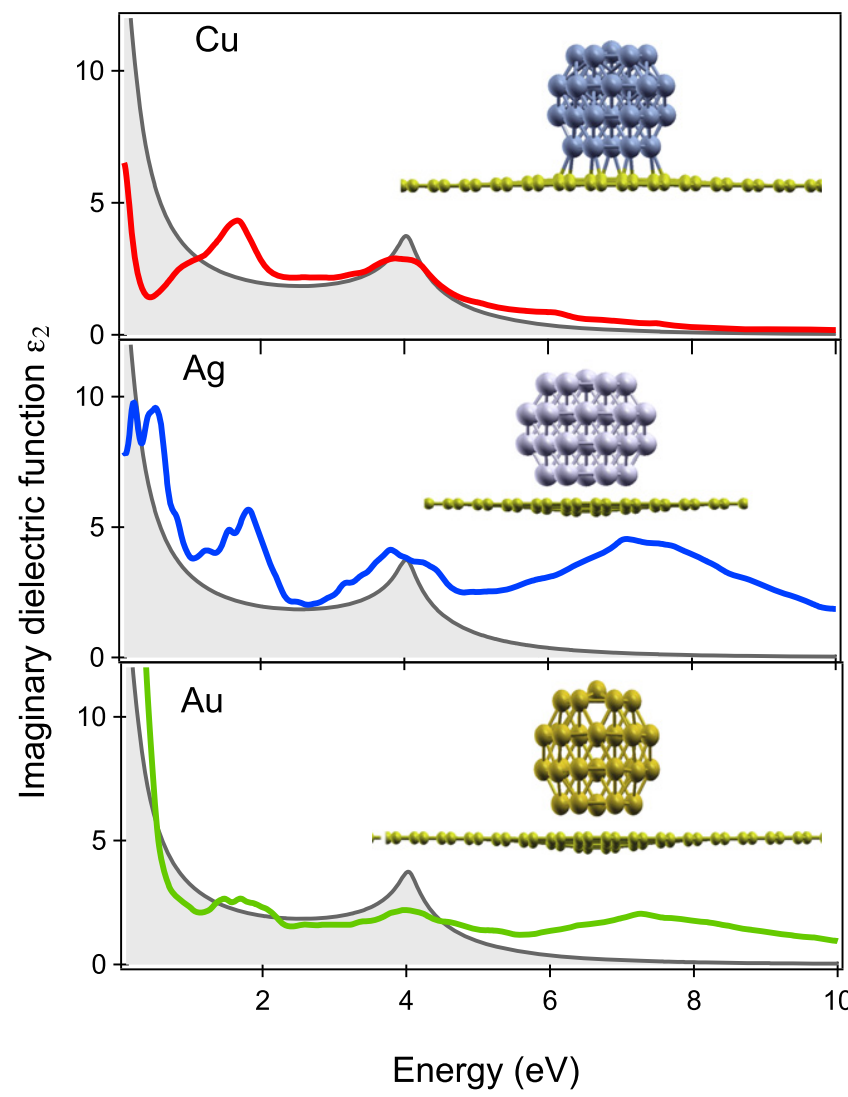

Figure 4. Comparison between the imaginary part of the dielectric functions $\epsilon_{2}$ calculated for graphene decorated with $\mathrm{Cu}$ (red), $\mathrm{Ag}$ (blue) and $\mathrm{Au}$ (green) clusters of $0.8 \mathrm{~nm}$ in diameter. For comparison, the dielectric function $\epsilon_{2}$ for pristine graphene is shown (gray shaded).

time, we infer that the larger photo-response measured on $\mathrm{Cu}$ decorated CNT can be partially justified by the higher intensity and by the longer relaxation time of the second dynamics present in the transient optical response of the decorated CNT.

\section{Conclusion}

Time-resolved reflectivity on MWCNTs, bare and decorated with $\mathrm{Au}, \mathrm{Ag}$ and $\mathrm{Cu}$ nanoparticles, has been investigated.

A Drude-like behavior of the photoexcited carriers, observed on the bare MWCNTs, persists even when the CNT wall is decorated with metallic NPs. The presence of the NPs enhances the Drude-like photoabsorption response of the CNTs thanks to a static charge transfer from the NPs to the tubes. The highest efficiency of this phenomenon has been observed for the sample with $\mathrm{Cu}$ NPs.

In addition to the fast dynamics, a second slow dynamics involving electronic states localized at the CNT-NP interface appears in decorated samples.

The results reported here corroborate the statement that transient optical spectroscopy, giving insight into the photocarrier generation mechanism, is a powerful tool to investigate the relaxation dynamics in carbon-based hybrid systems 
and allows us to improve in a targeted way the performance of the devices based on such hybrid systems.

\section{Acknowledgments}

MS and PC thank Professor F Nanni from the University of Tor Vergata for the SEM measurements and acknowledge the financial support from the Air Force Office of Scientific Research Material Command, USAF, under Grant No. FA8655-11-1-306. OP thanks CINECA for granting CPU time.

LM acknowledges financial support from the EC ITN project CLERMONT4 (Grant No. 235114).

SP thanks the MIUR for supporting this work under Contract No. PRIN 2010BNZ3F2 and acknowledges partial support from D.2.2 grants of the UCSC.

OP and MS acknowledge EU funding within the HORIZON2020 RISE project CoExAN (GA 644076).

\section{References}

[1] Baughman R H, Zakhidov A A and De Heer W A 2002 Science 297 787-92

[2] Scarselli M, Castrucci P and De Crescenzi M 2012 J. Phys.: Condens. Matter 24313202

[3] Robel I, Bunker B A and Kamat P V 2005 Adv. Mater. 17 2458-63

[4] Spataru C D, Ismail-Beigi S, Benedict L X and Louie S G 2004 Phys. Rev. Lett. 92 774021-4

[5] Perebeinos V, Tersoff J and Avouris P 2004 Phys. Rev. Lett. 92 257402-1

[6] Huang L and Krauss T D 2006 Phys. Rev. Lett. 96057407

[7] Gabor N M 2013 Acc. Chem Res. 46 1348-57

[8] McEuen P L and Park J-Y 2004 MRS Bull. 29 272-5

[9] Guldi D M, Rahman G M A, Zerbetto F and Prato M 2005 Acc. Chem Res. 38 871-8
[10] Wildgoose G G, Banks C E and Compton R G 2006 Small 2 182-93

[11] Rutherglen C, Jain D and Burke P 2009 Nat. Nanotechnol. 4 811-9

[12] Kong J, Franklin N R, Zhou C, chline M G, Pengn S, Cho K and Dai H 2000 Science 287 622-5

[13] Goldoni A, Petaccia L, Lizzit S and Larciprete R 2010 J. Phys.: Condens. Matter 22013001

[14] Zanolli Z, Leghrib R, Felten A, Pireaux J-J, Llobet E and Charlier J-C 2011 ACS Nano 5 4592-9

[15] Rigoni F, Drera G, Pagliara S, Goldoni A and Sangaletti L 2014 Carbon 80 356-63

[16] Eder D 2010 Chem. Rev. 110 1348-85

[17] Kamat P V 2007 J. Phys. Chem. C 7 2834-60

[18] Zhu X Y, Yang Q and Muntwiler M 2009 Acc. Chem. Res. 11 1779-87

[19] Jia Y, Wei J, Wang K, Cao A, Shu Q, Zhu Y Gui X, Zhuang D, Zhang G, Ma B, Wang L et al 2008 Adv. Mater. 20 4594-8

[20] Gabor N M, Zhong Z, Bosnik K, Park J and McEuen P L 2009 Science 325 1367-71

[21] Scarselli M, Camilli L, Matthes L, Pulci O, Castrucci P, Gatto E, Venanzi M and De Crescenzi M 2012 Appl. Phys. Lett. 101241113

[22] Scarselli M, Camilli L, Castrucci P, Nanni F, Del Gobbo S, Gautron E et al 2012 Carbon 50 875-84

[23] Kohn W and Sham J 1965 Phys. Rev. 140 A1133

[24] Hohenberg Kohn W 1964 Phys. Rev. 136 B864

[25] Ceperley D M and Alder B J 1980 Phys. Rev. Lett. 45566

[26] Perdew J P and Zunger A 1981 Phys. Rev. B 235048

[27] Giannozzi P, Baroni S, Bonini N, Calandra M, Car R, Cavazzoni C, Ceresoli D, Chiarotti G L, Cococcioni M, Dabo I et al 2009 J. Phys.: Condens. Matter 21395502

[28] Ma Y-Z, Hertel T, Vardeny Z V, Fleming G R and Valkunas L 2008 Ultrafast Spectroscopy of Carbon Nanotubes (Topics in Applied Physics vol 111) (Berlin: Springer) pp 321-52

[29] Seibert K, Cho G C, Kutt W, Kurz H, Reitze D H, Dadap J I, Ahn H, Downer M C and Malvezzi A M 1990 Phys. Rev. B 42 2842-51

[30] Galimberti G, Pagliara S, Ponzoni S, Dal Conte S, Cilento F, Ferrini G, Hofmann S, Arshad M, Cepek C and Parmigiani F 2011 Carbon 49 5246-52

[31] Link S and El-Sayed M A 2003 Annu. Rev. Phys. Chem. 54 331-66 